\title{
Research on supplier selection model based on an improved TOPSIS
}

\author{
Zhen GU, Yu WANG, Lu CAO \\ Department of Management, Jincheng College, Nanjing University of Aeronautics \& \\ Astronautics ,China \\ goozhen123@163.com
}

Keywords: Improved TOPSIS method; Interval indicator; Suppliers' selection; Transform operator

\begin{abstract}
The traditional TOPSIS method to initialize the initial decision matrix are all used the linear transformation methods on [0,1] interval, these methods has the problem of "only award, no punishment". So this paper uses the principle of "reward good and punishment bad", puts forward a kind of "reward good and punishment bad" transform operator which is easy calculation and practical for benefit type v cost type and interval type decision indicators. Then it proposed an improved TOPSIS decision model. Finally this evaluation model is used to make an empirical study on the supplier selection problem, and the result of the empirical study verified the validity and feasibility of this method.
\end{abstract}

\section{INSTRUCTIONS}

TOPSIS method is the technique for order preference by similarity to an ideal solution, put forward by C.L.Hwang and K.Yoon in 1981. TOPSIS is the method that is sorted according to the proximity of the limited object and ideal goal, is to evaluation the relative merits and disadvantages of the object.

The basic idea of TOPSIS is: set a virtual optimal solution (known as the ideal solution) and a virtual the worst solution (known as the negative ideal solution), in the target space solution calculate the relative approach degree, to measure a solution close to the ideal solution and far from negative ideal solution, with relative proximity values decide on the sort and relative proximity The greater the degree of relative proximity, the more optimal scheme, the contrary, the worse.

TOPSIS method is an effective method in MADM,(Multiple Attribute Decision Making), and is widely used in the field of economic management, etc.

The research of supplier selection method has experienced three development stages: qualitative method, quantitative method, combination qualitative and quantitative method. Early supplier selection method used qualitative methods, it mainly based on experience and relationship with suppliers to make subjective judgment. However, due to the lack of scientific basis and subjectivity, this method is gradually abandoned by purchasing managers. But in some small companies this method is still widely exist, with the benefit of simple operation, save costs and so on. Then, using quantitative methods to select suppliers has gradually been recognized and become an important part of research and application. However, with the development of research and application, it is found that there are many factors that affect the selection of suppliers, there are not only quantitative factors, but also have many qualitative content, only using the quantitative method has been unable to meet the actual needs and often faces the problem when price, delivery, quality and ability conflicted with each other. Therefore, the research to the supplier selection theory and method turns to the stage of combination qualitative and quantitative method. It is agreed that the supplier selection is a multi objective or multi attribute decision making problem. It is a general trend to use the multi objective decision theory which has been developed or is developing to solve the problem of supplier selection. At present, the quantitative model for supplier selection are AHP method, fuzzy comprehensive evaluation method, and neural network method, TOPSIS method, data envelopment analysis (DEA), linear programming method and so on.

Based on the traditional TOPSIS method, this paper proposed a kind of "reward good and punishment bad" transformation operator which is easy calculation and practical. Then an improved 
TOPSIS decision model it established. Finally, this improved model is used to a make an empirical analysis of the supplier selection problem.

\section{ESTABLISHMENT OF THE TOPSIS DECISION MODEL}

Assume multiple criteria decision making problem has $n$ evaluation objects or decision scheme, and the decision scheme set $S$ is composed of these evaluation objects or decision scheme, $\mathrm{S}=\left\{S_{1}, S_{2}, \Lambda, S_{n}\right\}$; the indicator set $A$ is composed of $m$ evaluation indicator or attribute, $\mathrm{A}=\left\{A_{1}, A_{2}, \cdots, A_{m}\right\}$; The effect sample values of the scheme $S_{i}$ on the indicator $A_{j}$ is $x_{i j}(i=1,2, \Lambda, n ; j=1,2, \Lambda, m)$. Then the decision matrix of the decision scheme set $S$ on the indicator set $A$ is expressed as

$$
X=\left[\begin{array}{cccc}
x_{11} & x_{12} & \cdots & x_{1 m} \\
x_{21} & x_{22} & \cdots & x_{2 m} \\
\cdots & \cdots & \cdots & \cdots \\
x_{n 1} & x_{n 2} & \vdots & x_{n m}
\end{array}\right]
$$

According to different natures of all the indicators, the indicator set $\mathrm{A}=\left\{A_{1}, A_{2}, \cdots, A_{m}\right\}$ can be divided into three types, "benefit type" indicator, "cost type" indicator and "interval type" indicator. The "benefit type" indicator is the indicator that its value is the bigger the better; the "Cost type" indicator is the indicator that its value is the smaller the better; the " interval type " indicator means that its value is the better if its value is in a given interval $[A, B]$.

\section{1 "Reward good and punishment bad" transform operator}

Because of the different dimensions of the indicator, it is difficult to compare them directly in the decision-making process, so it is necessary to initialize the original effect sample matrix. But using different initialization transformation processing methods may lead to different evaluation results. And the existing methods to initialize the initial decision matrix are all used the linear transformation methods on [0,1] interval, these methods has the problem of "only award, no punishment". So this paper uses the principle of "reward good and punishment bad", puts forward a kind of "reward good and punishment bad" transform operator which is easy calculation and practical. The basic idea of this transform operator is: if the indicator value of the evaluation object is better than average, given the positive value of 0 to 1 , worse than average, given the negative value of -1 to 0 .

If $A_{i}$ is a benefit indicator:

$$
r_{i j}=\left\{\begin{array}{cc}
\frac{x_{i j}-x_{i}}{\max _{i}\left\{x_{i j}\right\}-x_{i}} & \max _{i}\left\{x_{i j}\right\} \neq x \\
1 & \max _{i}\left\{x_{i j}\right\}=x
\end{array} \quad i=1,2, \cdots n\right.
$$

$X_{i}$ is the effect critical value of $i$ indicator (the effect critical value can be determined according to the actual decision situation) .

If $A_{i}$ is a cost indicator:

$$
r_{i j}=\left\{\begin{array}{cl}
\frac{x_{i}-x_{i j}}{x_{i}-\min _{i}\left\{x_{i j}\right\}} & x_{i} \neq \min _{i}\left\{x_{i j}\right\} \\
1 & x_{i}=\min _{i}\left\{x_{i j}\right\}
\end{array} \quad i=1,2, \cdots n\right.
$$

$X_{i}$ is the effect critical value of $i$ indicator (the effect critical value can be determined according to the actual decision situation) .

For the interval indicator (including fixed indicator, at this point $A=B$ ): 


$$
r_{i j}= \begin{cases}1-\frac{A-x_{i j}}{\max \left\{\max _{i}\left\{x_{i j}\right\}-B, A-\min _{i}\left\{x_{i j}\right\}\right\}}, & x_{i j} \leq A \\ 1, & \ldots A \leq x_{i j} \leq B \cdots i=1,2, \cdots n \\ 1-\frac{x_{i j}-B}{\max \left\{\max _{i}\left\{x_{i j}\right\}-B, A-\min _{i}\left\{x_{i j}\right\}\right\}}, & B \leq x_{i j}\end{cases}
$$

The above $r_{i j}$ is called "reward good and punishment bad" transform operator.

By using this transform operator, the sample matrix $X$ is transformed into the decision matrix $R$.

$$
R=\left[\begin{array}{cccc}
r_{11} & r_{12} & \cdots & r_{1 m} \\
r_{21} & r_{22} & \cdots & r_{2 m} \\
\cdots & \cdots & \cdots & \cdots \\
r_{n 1} & r_{n 2} & \vdots & r_{n m}
\end{array}\right]
$$

The elements of the matrix $R$ are dimensionless, all the elements are in line with the "reward good and punishment bad" standard, and for arbitrary $r_{i j}$,

$$
r_{i j} \in[-1,1],(i=1,2, \cdots, n ; j=1,2, \cdots, m)
$$

\subsection{The steps of TOPSIS algorithm}

Step 1: according to the multiple criteria decision-making problem construct effect sample matrix $X=\left(x_{i j}\right)_{n \times m}$.

Step 2: using the formula of (1), (2), (3) transform the effect sample matrix $X$ into the decision matrix $R$.

Step 3: determining the weight vector matrix $w$ of each indicator by the AHP method, $w=\left(w_{1}, w_{2}, \Lambda, w_{m}\right)$.

Step 4: constructing normalized weighted decision-making matrix $Z$.

$$
Z=\left(z_{i j}\right)_{n m}=R * w=\left[\begin{array}{cccc}
w_{1} r_{11} & w_{2} r_{12} & \cdots & w_{m} r_{1 m} \\
w_{1} r_{21} & w_{2} r_{22} & \cdots & w_{m} r_{2 m} \\
\cdots & \cdots & \cdots & \cdots \\
w_{1} r_{n 1} & w_{2} r_{n 2} & \vdots & w_{m} r_{n m}
\end{array}\right] \text {. }
$$

Step 5: determining the reference sample

Using the best value and the worst value of each indicator constitute the best value vector $Z^{+}$, the worst value vector $Z^{-}$.

$$
Z^{+}=\left(z_{1}^{+}, Z_{2}^{+}, \Lambda, z_{m}^{+}\right) \text {; }
$$

$$
Z^{-}=\left(z_{1}^{-}, z_{2}^{-}, \Lambda, z_{m}^{-}\right)
$$

And: $z_{j}^{+}=\max \left(z_{1 j}, z_{2 j}, \Lambda z_{n j}\right), j=1,2, \Lambda m$

$$
z_{j}^{-}=\min \left(z_{1 j}, z_{2 j}, \Lambda z_{n j}\right), j=1,2, \Lambda m
$$

Step 6: calculating the distance between the scheme and the optimal value and the worst value respectively.

$$
D_{i}^{+}=\sqrt{\sum_{j=1}^{m}\left(z_{i j}-z_{j}^{+}\right)^{2}}, \quad D_{i}^{-}=\sqrt{\sum_{j=1}^{m}\left(z_{i j}-z_{j}^{-}\right)^{2}}
$$

Step 7: calculating the relative approach degree

$$
C_{i}=\frac{D_{i}^{-}}{D_{i}^{+}+D_{i}^{-}}, \cdots i=1,2, \cdots n, \quad 0 \leq C_{i} \leq 1
$$

Step 8: sorting scheme.

According to the value of $C_{i}$ sort each scheme, the greater the value of $C_{i}$, the better of the 


\section{EMPIRICAL ANALYSIS}

In order to verify the feasibility of the above model, an empirical about supplier selection is analyzed and calculated in this paper.

Supplier selection is related to many factors, and has a variety of evaluation index, sometimes even contradictory, combination of qualitative method with quantitative, the weight of each index is not the same, not only need to reflect the service index including quality, delivery time, price and so on, but also need to reflect the supplier's service level and competitiveness in expanding index, and these all can be able to evaluate suppliers comprehensively, comprehensively, objectively and scientifically.

(1) The principle of wholeness. Evaluation index should be set up to evaluate the various aspects of the object, and rational structure the number of the indicators. In this way, we can scientifically reflect the object of evaluation, so as to correctly express the purpose of evaluation. How to divide the index does not have an absolute objective standard, and should be determined according to the actual situation. Selection of evaluation indicators, it is necessary to consider the positive income, also need to consider the negative risk, only a full range of indicators can ensure the integrity of the content of the evaluation.

(2) The principles of emphasis. In the selection of evaluation index, may not share everything. If all indicators lists together, neither highlighting comprehensive index in the evaluation of leading role, but also emphasizes the influence degree of some specific indicators of evaluation, resulting in no clear logic judgment, primary and secondary is unknown, and it is difficult to achieve fully and accurately reflect the basic goal of supplier selection. To filter as far as possible the most important indicators associated with the target, set the number of indicators to be as simple as possible and summarized, the secondary indicators can be properly rough.

(3) The principles of comparative. Index system should reflect the commonality of all the evaluated enterprises, the data should be used to quantify the qualitative indicators as far as possible, the quantify the data of qualitative indexes can be given by the expert or Delphi method, and to ensure the comparability of evaluation results.

(4) The principles of maneuverability. The index system needs some flexibility, which can reflect the enterprise's own characteristics and the actual situation.

(5) The principles of measurable. When selecting the key performance indicators, it needs to follow the principle of measurable. The index itself or quantitative, or behavior, these index's verification data and information can be obtained, easy measurement and definition. In addition, the data should be collected easily, and the calculation is simple.

(6) The principle of expanding. The index system is not only to adapt to the future development, but also can selectively use some of the index system in accordance with the actual situation of enterprises.

In this case, the supplier evaluation index system is designed based on the goal of maximizing the supply chain performance and the five main principles, as shown in figure 1 .

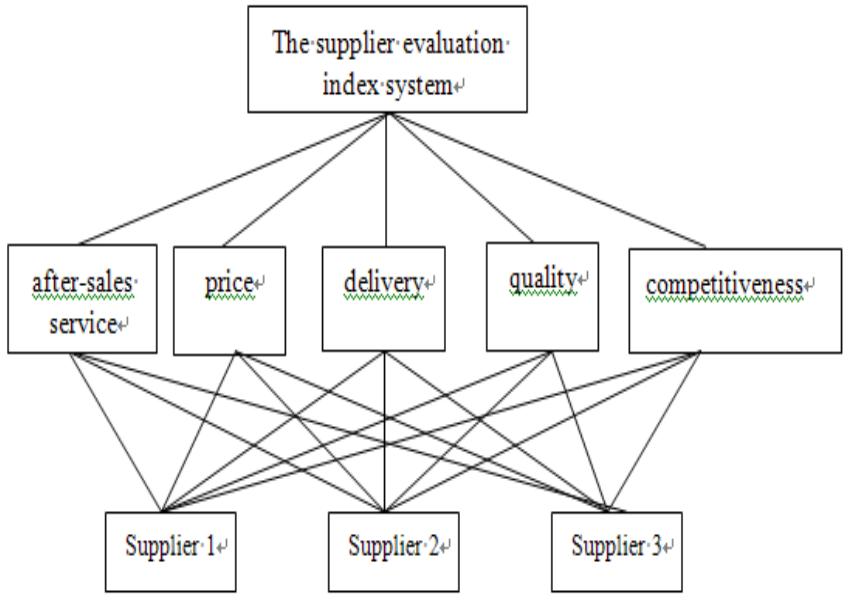

Figure 1. The supplier evaluation index system

In this index system three potential suppliers were evaluated by 5 indicators, including quality, price, delivery, after-sales service and competitiveness, then we can get the decision matrix $X$. 


$$
X=\left[\begin{array}{ccccc}
9.5 & 14.2 & 15.5 & 9.6 & 9.5 \\
9.4 & 15.1 & 17.5 & 9.3 & 9.7 \\
9 & 13.9 & 19 & 9.4 & 9.2
\end{array}\right]
$$

Among the 5 indicators, the competitiveness, quality, after-sales service are efficiency indicator, the greater the value, the better, the critical value is $x_{i}=15, i=2$. The price indicator is cost indicator, the lower the price, the better, the critical value is $x_{i}=15, i=2$; The delivery indicator is interval indicator, taking the special value $A=B=16$. Then the decision matrix $R$ can be get by using the formula (1)、(2) and (3)

$$
R=\left[\begin{array}{ccccc}
1 & 0.727 & 0.833 & 1 & 0.714 \\
0.8 & -0.091 & 0.5 & 0.5 & 1 \\
0 & 1 & 0 & 0.667 & 0.286
\end{array}\right]
$$

Determine the weight of each target and the corresponding indicators by the AHP method, as shown in Table 1.

\begin{tabular}{ccc}
\hline $\begin{array}{c}\text { Evaluating } \\
\text { indicator }\end{array}$ & $\begin{array}{c}\text { serial } \\
\text { number }\end{array}$ & weight \\
\hline quality & 1 & 0.25 \\
price & 2 & 0.22 \\
delivery & 3 & 0.18 \\
after-sales & 4 & 0.18 \\
service & 5 & 0.17 \\
competitiveness & 5 & \\
\hline
\end{tabular}

Table 1. The weight of each evaluating indicator and the corresponding indicators Then normalized weighted decision matrix $Z$ can be get.

$$
Z=\left[\begin{array}{ccccc}
0.25 & 0.16 & 0.15 & 0.18 & 0.121 \\
0.2 & -0.02 & 0.09 & 0.09 & 0.17 \\
0 & 0.22 & 0 & 0.12 & 0.049
\end{array}\right]
$$

According to the formula (4), the best value vector $Z^{+}$and the worst value vector $Z^{-}$can be get. As follows:

According to formula (5)

$$
\begin{gathered}
Z^{+}=(0.25,022,0.15,0.18,0.121), \\
Z^{-}=(0,-0.02,0,0.09,0.049)
\end{gathered}
$$

$$
\begin{aligned}
& D^{+}=(0.077,0268,0.321) \\
& D^{-}=(0.362,0251,0.242)
\end{aligned}
$$

according to formula (6), the relative proximity can be calculated,

$$
C=\left(C_{1}, C_{2}, C_{3}\right)=(0.824,0.484,0.429)
$$

Because of $C_{1}>C_{2}>C_{3}$, it shows that the supplier $\mathrm{A}$ is the most competitive, so this company should choose the supplier $\mathrm{A}$ as its partner.

\section{CONCLUSIONS}

In this paper, an improved TOPSIS method is proposed by designing the "Reward good and punishment bad" transform operator. Finally this e improved TOPSIS model is used to make an empirical study on the supplier selection problem, and the feasibility of this method is proved. The results of this problem substantiate the feasibility of this method. 


\section{REFERENCE}

[1] Yaoguo Dang.2004. Decision model of multi indicator weighted grey target. Statistics and Decision (3):29-30.

[2] Sifeng Liu.2010. A new model of multi objective intelligent weighted grey target decision model Control and Decision25 (8) :1159-1163.

[3] Liyan Zhou.2011. Evaluation and optimization of equipment procurement contractor based on improved TOPSIS method[J]. Logistics Technology (3) :112-116.

[4] Xiaojian Wang.2009. Research on comprehensive evaluation of electronic commerce website based on improved TOPSIS method. Electronic Commerce53 (53):129-132.

[5] Jing FU.2010. Research on supplier evaluation model based on improved TOPSIS method. Modern Business (12):164-166.

[6] Wen-kun ZHOU.2005.A Method for Supplier Selection Based on Improved TOPSIS. Operations research and manageme nt science14(6):39-44. 